i.e., wherever the blood circulates; and, therefore, on the principle of treatment adopted in this case by Dr. Davies-viz., by the process of its eliminationit is assuredly as rational to encourage the elimination per vias naturales, that is, by the sudoriparous glands, as it is through the serum of the blistered surfaces of the inflamed joints. The serum of even many blistered joints must in amount be very greatly less than the perspiration morbidly excited by the disease, and actively encouraged by artificial means, from the whole cutaneous surface of a rheumatic patient.

The application of blisters to the joints, as recommended by Dr. Davies, may be certainly considered a good and rational proposition in the treatment of acute rheumatism, and will, no doubt, on his recommendation, be fully tried. But it should surely, at present, at all events, be only regarded as an adjunct to other and far more important treatment. To regard it, as Dr. Davies seems to do, as the primum mobile in the cure-as a sort of specific methodus curandi of acute rheumatism-is what I have here ventured to object to in my remarks upon Dr. Davies's papers.

\section{ON CHOREA, AND ON NATURE AND ART IN THE TREATMENT.}

By Jalies Turnbulu, M.D., Physician to the Liverpool Royal Infirmary.

[Read before the Liverponl Medical Society, December 1st, 1964.]

Chorea, or St. Vitus's Dance, is well known as a nervous affection of spasmodic nature, manifesting itself by irregular contractions or twitchings of the voluntary muscles, which the patient cannot control. It occurs much more frequently in females than in males; and more commonly between the ages of 8 and 15 than in younger children or grown-up persons. Those attacked are not usually of strong constitution, but of nervous temperament and sensitive disposition; and fright is often the exciting cause. $I$ have observed that in females it occurs most frequently just before the catamenial function is established; and, when it comes on after this, there is generally some menstrual irregularity, accompanied with more or less anæmia. I have also observed the occurrence of worms in connection with the disease; but intestinal irritation has not often appeared to me to be the sole cause.

The connection between chorea and rheumatism is one of the most singular facts in relation to this disease, and I have had many opportunities of observing it in private, as well as in hospital practice. We frequently see chorea coming on while the joints are swelled and painful from an attack of acute or subacute rheumatism, and it appears in such cases to supersede the rheumatic affection. Again, we see cases of chorea where the joints become swelled and painful from rheumatism, supervening or recurring. In either case, the one disease seems to take the place of the other. Besides this, we find that chorea occurs frequently in families where there is the rheumatic diathesis, one member being attacked with chorea, and others liable to rheumatic attacks. Chorea is a functional disorder, and in fatal cases no organic alterations which could be the cause of the disease have been discovered, but the organic effects which rheumatic fever prodaces on the heart and its valves have been not unfrequently observed. As we have good reason to believe that rheumatism is produced by a materies morbi in the blood, the close connection between rheumatism and chorea suggests the inquiry whether the latter might not be dependent on the same or a similar cause. Some countenance is also given to such a theory, by the fact that when urea is retained in the blood in consequence of disease of the kidneys, there is a liability to convulsive and other nervous affections, and also by the fact that some poisons, such as strychnia, when introduced into the blood, excite spasm of the muscles.

With respect to the relation in which chorea stands to other diseases of the nervous system, I would observe that it bears to paralysis this relation, that we sometimes find in chorea not only that the patient is unable to use the muscles of the tongue so as to speak, but that it is impossible to use some of the limbs, owing to a kind of temporary imperfect paralysis. It bear's also to paralysis this further relation, that in some cases one side only of the body is affected. Epilepsy is a more intractable and severe disease, at tended with protracted muscular spasm and complete loss of consciousness, and ultimate injury of the mental powers, which never occur in chorea; but the absence of any traceable organic alteration of the brain or cord characterises both. As regards severity, chorea holds an intermediate place between epilepsy and hysteria, the convulsive form of which latter is more troublesome than dangerous. I have seen some cases, however, where chorea and hysteria approximated so closely, that they might not inappropriately be called cases of hysterical chorea.

Chorea is often a very trifling complaint, but it is also frequently a troublesome protracted disease, and in a few instances I have seen it prove fatal. It is then one of the most frightful diseases we can witness, causing violent contortions, and tossing the patient about in all directions, thus injuring and abrading all the prominent parts of the body, and causing rapid loss of flesh and death from exhaustion, owing to incessant motion and want of sleep. Whenever the patient sleeps, the twitchings cease, and thus the strength is renewed; but if the movements should be so violent as to preclude sleep, the prognosis becomes very grave.

The disease often hegins with slight twitching, and gradually attains its highest degree of severity; but very severe cases may recover more rapidly than slighter ones. I had two cases under my care at the same time this summer which illustrate this; one was a girl of 12 years of age, who was brought regulirly to see me at my house. Though the case was never very severe, it was much longer in recovering than the other, which was the most severe in which I have seen recovery take place. As to the duration of the disease, Dr. Hughes, in his digest of one hundred cases, tells us that, exclusive of the time the patients had been previously ill, it varied in Guy's Hospital from three weeks to three months, 40 per cent. of the cases being cured in from three to six weeks.

The general diffusion amongst medical men of the present day of a knowledge of the important fact that in all curable diseases there is a spontaneous tendency to recovery, is a distinguishing feature of our times ; and, when combined with a due appreciation of the influence of art in promoting this object, such knowledge must exercise a most beneficial influence on practice. Sir John Forbes's little treatise is the only one in which we have a comprehensive examination of this subject, and whilst I wouid observe that I place a high value on the service which he rendered by his exposition of the power of nature in the cure of disease, I must add that I think the tendency of his work is to repress, and unduly repress, the progress of medicine in the direction of therapeutic inquiry. 
I feel convinced, too, that if Jenner had not had a higher appreciation than this author of the powers of art, he would never have traced the operations of nature so as to make his grand discovery of the preventive power of vaccination, in which we have a beautiful illustration of art searching out and applying one of the powers of nature.

Like other curable diseases, chorea shows a natural tendency to spontaneous recovery; and Dr. Wilks, having treated some cases by diet and regimen alone, has advocated expectant treatment; and in a clinical lecture which he published, has endeavoured to show that other treatment is of comparatively little importance. "Where a malady," he observes, "can be cured by so many remedies, it may be very well left to itself, and can be placed in the same category as hooping-cough, and many other disorders which tend to a spontaneous cure." I would observe, however, that not only in disorders, but in all curable diseases, there is the same tendency to spontaneous cure. Without the assistance which has been known as the vis medicatrix nature, the physician would be powerless; and those are undoubtedly the safest and best practitioners who are the closest observers of nature's operations, and the most successful assistants in aiding her in her own methods of effecting recovery. But this principle applies as much to serious and acute diseases as to such nervous disorders as chorea and hooping-cough; and the tendency to spontaneous recovery may be quite as well, or better illustrated by referring to such acute diseases as pneumonia, the exanthematous ferers, and ague.

In the time of Sydenham, inflammation of the lungs and chorea were both treated by bleeding; and we know that in the former it was till a comparatively recent period repeated again and again coup sur coup, as if the only object of the medical man had been to wage war with the disease, instead of it being used as a means to promote the natural process of recovery-the only way in which it can ever be admissible as a therapeutic agent in this disease. Repeated bleeding we in the present day consider a violent, injurious, and unscientific interference with nature's operations its moderate use may no doubt be so applied in some cases as to promote indirectly the natural tendency to recovery; but practically we know now that the great majority of cases of simple pneumonia may be far more safely and successfully treated by mild means-by eliminating and sustaining treatment, having for its object the promotion of the natural tendency to spontaneous recovery. In all the acute infectious diseases, we can as yet only assist nature in effecting a spontaneous cure, but there is a wide field here for the practical sagacity of the medical man in removing obstacles, and assisting her operations.

Ague is another disease in which we observe the same tendency to spontaneous recovery. Cases admitted into an hospital would in this country generally recover under the influence of diet, regimen, and warmth, just as in many cases of chorea. We do not, however, trust to these alone, for we know that in quinine we have a remedy which rapidly cures the disease, or, it may be, only promotes effectively the natural tendency to spontaneous recovery.

It would be easy to carry this comparison further; but let us turn to the practical question, whether we are, because many cases of chorea would recover by treatment by regimen only, to stand by and watch the case merely. The clinical observations of Dr. Wilks, to which I have referred, undoubtedly tend to this result, and are calculated to depreciate therapeutic inquiry, and thus to paralyse the search for more efficient remedies. "It is well known," he observes, "that there are fifty remedies against this disorder, and even more than this number may be discovered, if it be worth while to collect them from medical writings." These and other remarks were made with reference to sulphate of aniline, introduced by me; and it is to be regretted that attempts should thus be made to depreciate therapeutic inquiry, which is one of much difficulty. In replying to the general question, whether we should merely watch the disease or not, I would express my opinion that, whilst we should in this, as in all other diseases, avail ourselves of the spontaneous tendency to cure, we may render valuable assistance by remedial means, and that such aid is often urgently needed on account of the disease being severe or protracted. Dr. Wills does not, however, although an advocate of expectant treatment, entirely deny the efficacy of remedies; for he says: "It would appear to be true that tonics are useful, and that it is important to correct any morbid state of the alimentary canal." The mineral tonics, such as the preparations of iron, are undoubtedly the most generally useful remedies in ordinary cases of the disease; but where the usual means have failed, I have so often seen the disease removed by the direct sedative influence of sulphate of aniline, that I would still recommend a trial of it in such cases.

The following case is interesting, both as an illustration of what may be termed hysterical chorea, and as affording an example of successful treatment by this remedy, whether such result may be considered to have been due to its direct sedative influence on the nervous system, or partly to the mental impression it produced on the patient-an influence in the treatment of such cases which should not be overlooked.

A lady, aged about 25, was placed under my care on November 16th, 1861, on account of a peculiar nervous affection, from which she had suffered more or less for eight years. In the early period of her illness, she had been under the care of the late Sir B. Brodie, and had afterwards been under the treatment of several irregular practitioners, but without receiving permanent benefit. The nervous affection consisted of an inability to control the muscles of the right arm, and in a slighter degree those of the left. There was an almost constant contraction of the muscles of the right shoulder and arm, causing a painful fidgeting motion, and compelling her to hold the arm with the left hand. There was plenty of muscular power; but she could not regulate the movements so as to write or do any kind of work. In walking, she had not perfect control of her movements, and exhibited some of the peculiar jerking seen in St. Vitus's dance. As regarded the general symptoms, the complexion was rather anæmic; the muscular development good; the tongue natural; the digestion pretty good; the bowels sluggish; the pulse quick; and menstruation regular, but scanty. The treatment was first directed to the improvement of the general health by the preparations of iron combined with ammonia, and by out-door exercise, the use of dumb-bells, etc. Warm hip-baths, with mustard, were given; sedative embrocations, with atropia, etc., were used to the arm and shoulder; and aloetic aperients were administered. By these means, the general health was improved; but no influence was produced on the nervous disorder.

On the 10th of January, after she had been nearly two months under the previous treatment, I prescribed sulphate of aniline in three-grain doses thrice a day. It produced blueness of the lips and face more rapidly than in any other case $I$ have seen; and, in other respects, she seemed peculiarly sensitive to the influence of the remedy, which always produced in the evening, after the third dose, not only decided 
blueness, but also nervous depression, discomfort at the epigastrium, slight headache and palpitation, quickness of pulse, and coolness of the hands. At the same time, it had a marked beneficial influence on the disease; the muscular irritability was rapidly diminished, and she regained the control of the right hand and arm. The depressing effect of the remedy made it necessary to watch carefully its action, and to reduce the quantity to two doses daily. It was continued for three weeks, with occasional intermission for a day; and she had then completely regained the control of all her muscles, and could play on the piano. The blueness in this, as in all cases, went off within twenty-four hours after laying aside the remedy. About the middle of February, she had some return of the nervous symptoms, which soon yielded to the same treatment ; and, at the end of the month, she returned home quite well.

Aniline has a powerful influence on the nervous system when taken internally, and still more when inhaled. From being accidentally inhaled, it has in several instances produced serious poisonous effects, though it has not been known to cause fatal effects on man. In recommending it as a remedy in chorea, I advised that the sulphate should be given in doses of two or three grains; and that it should be diminished or omitted when it produced the depressing effect, which is usually experienced in proportion to the temporary blueness of the surface it causes.

Dr. Frazer, who published some cases in which he gave it unsuccessfully, appears to have administered it in a different way-in large and increasing doses, as sulphate of zinc has sometimes been given-and as if his object had been to test its toxic as much as its therapeutic effects. In one of his cases, he tells us that the patient took twenty-eight grains daily. In thirty-four days, the quantity taken was four hundred and six grains; and it was continued all this time, although it caused frontal pain and aggravated deafness. "The result," he observes, "points nevertheless to the inefficacy of sulphate of aniline in five cases ; and is, therefore, pro tanto, a clinical fact to be noted." I believe that any agent which produces a violent impression on the nervous system may prove injurious in this disease; and I advised the employment of aniline only so as to produce a gentle sedative influence; and, as mot one out of five cases thus treated in hospital improved or recovered under this mode of treatment from a disease which Dr. Wilks has shown that many patients get well of under regimen only, the result would seem to show further, that thus given, as an antidote against the disease, it may prove not merely inefficacious, but injurious.

Having referred to the toxic properties of aniline, I would here observe that they have been erroneously regarded as of similar character with those of a more highly narcotic agent-nitro-benzine-which has very noxious properties, but has nevertheless been much used for flavouring and in perfuming, on account of its resemblance to the oil of bitter almonds. The efiects of aniline and nitro-benzine have been ccnfounded together from these circumstances. When aniline is introduced into the system, it undergoes oxidation, by which the mauve colouring matter is produced, as I was the first to point out. When nitro-benzine is introduced either through the stomach or by inhalation of the vapour, a portion is first converted into aniline, and this again undergoes further change, so that the same blueness is caused by both. The effects of nitro-benzine are, however, essentially different, and more persistently narcotic than those of aniline, as has been shown by Dr. Taylor in Guy's Hospital Reports, in a recent paper in which he has fully examined the various cases in which the toxic effects of these agents have been known to be produced; and he has recognised the difference in the effects of aniline and nitrobenzine. There is one fact, however, which appears to have escaped attention. It is this : that nitrobenzine is one of a series of chemical compounds which have similar effects on the nervous system. They are the xyloids-a series of substitution compounds formed by the action of nitric acid on starch (xyloidine), on sugar.(saccharoine), on cotton (pyroxiline or gun-cotton), on glycerine (glonoine or nitroglycerine), and on benzine (benzoine or nitro-benzine) Organic compounds containing nitrogen have often a powerful influence on the animal economy, as is the case with the vegetable alkaloids; and these are all nitrogenous compounds of similar chemical constitution.

Dr. Edwards, in a paper on the Physiological Properties of Xyloids, published in the Liverpool MedicoChirurgical Journal, first drew attention to the action of these agents, and showed that they have similar and peculiar effects on the nervous system : the amount of their action being, in a measure, proportioned to their solubility. "They all appear" (he says) " to possess, more or less, the peculiar action on the nervous system characteristic of strychnine; and I believe that further experiments on men would prove that they possess peculiar and valuable medicinal properties. Physically, they are allied; being all highly explosive bodies, deflagrating at a low temperature or by concussion; and physiologically, they all produce a powerful effect on the heart's action, trismus, and a series of tetanic convulsions, terminating in death."

Having witnessed most of Dr. Edwards's experiments, I have taken this opportunity, of not only pointing out that nitro-benzine is different in its effects from aniline, but that it is one of the xyloids, and that to Dr. Edwards the merit is due of first as certaining the poisonous properties of nitro-benzine, as well as its relation to the other xyloids.

Looking at chorea in a strictly practical point of view, I would now make a few remarks on the general treatment of the disease. In cancer, which is one of the most incurable diseases, many remedies have been, and will no doubt continue to be, tried; and in chorea, which is one of the most curable, nany remedies have likewise been tried. Niere number points, in reference to the first, to its intractable nature and, in regard to the second, to the fact that, though generally a curable disease, none of the remedies in use can be relied on to control the disease with any certainty. Indeed, practically, we know that there are cases which for months, and even years, resist both judicious and expectant treatment; and, therefore, the study of therapeutics in reference to this disease is still worthy of attention.

The remedies which have been chiefly used are, purgatives; the mineral tonics-iron, zinc, and arsenic; cod-liver oil ; electricity; and the showerbath. Purgatives I have given only when the state of the bowels rendered their use recessary ; and not, therefore, with the curative intention with which they have sometimes been administered. The mineral tonics uncoubtedly exert a beneficial influence. I do not believe they have any direct curative power; but they improve the general health, which is more or less below par, and thus they appear to lessen the excitability of the nervous system. Iron I believe to be the most generally useful, and especially in anæmic females. Dr. Hughes, in his digest of one hundred cases, makes these remarks :

"The different mineral tonics are very variously estimated by different practitioners, and one is often regarded as especially suited to the cure of chorea. 
While one practitioner regards arsenic as a specific for true chorea, another cures nearly all his cases with the sulphate or the oxide of zinc, and another considers the sulphate or the carbonate of iron as an almost infallible remedy. This may perhaps arise, in a great measure, from habit. It is possible that all may be nearly equally efficacious. I have seen each of these, and many other remedies cure the complaint; and 1 have seen them all occasionally fail.",

Zinc has been given in large doses, especially at Guy's Hospital; where Dr. Hughes has stated that thirty-six grains of the sulphate have been administered daily. It appears such an abuse of medicine, to prescribe these enormous doses of an irritant drug commonly used in such quantities as an emetic, that we are at loss to discover how it should ever have been thus given. What Sir John Forbes has termed the autocracy of Nature, could not have been understood by those prescribing it in such a manner; and Dr. Hughes observes :

"In the practice of the hospital, I am not aware that it has been shown that small or moderate doses of the mineral, continued an equally long time, will not effect a cure."

In my own practice, I have used iron and arsenic more frequently than zinc ; and I consider that, of the metallic tonics, iron is the best, and that arsenic stands next to it.

Cod-liver oil is a remedy which is sometimes of service, by improving the general health; and it may be given with, or subsequent to, the preparations of iron. Electricity seems, in some cases, to have acted beneficially, and in a very rapid manner.

The shower-bath is a remedial agent which gives tone to the system, and may be used along with other tonic treatment. I have seen it of the greatest use in many cases. In the case of a young lady, in whom the movements were incessant and violent, and prevented sleep, the movements subsided very much directly after the bath, and she soon fell asleep, and ultimately recovered. The emaciation and exhaustion of the patient from the incessant violent movements and the loss of sleep, had rendered the recovery of this patient very doubtful.

It is not less important to glance now at the means which may prove detrimental, and which should therefore be avoided in the treatment of this nervous affection. All violent impressions on the nervous system should be guarded against. We know that fright is not an unfrequent cause of the disease. It is probably on this account that the shower-bath may prove injurious when injudiciously employed, as well as very frequently useful. Narcotic remedies, such as opium, morphia, and hyoscyamus, have appeared to me (contrary to what might be expected) to be not simply useless, but positively hurtful. It has been said that chloroform is of use but I saw the inhalation tried in two very severe cases, which proved fatal; and though it produced a temporary sedative influence, it appeared to me to act injuriously on the general progress of the disease.

In all cases, due attention should be paid to hygienic as well as medicinal treatment, so as to improve in every way practicable the nutritive functions and the general health. The diet should be nutritious, and wine or malt liquors are often of service; and out-door exercise is desirable in the less severe cases. When the disease proves chronic, change of air is advisable; and in the summer the change may be made with advantage to the sea-side, where seabathing may be practised.

\section{ON THE PHYSICS OF DISEASE, AND THE PHYSICAL PATHOLOGY OF THE BLOOD.}

By Benjamin W. Richardson, M.A., M.D., Senior Physician to the Royal Infirmary for Diseases of the Chest.

\section{Chapter II.}

The Force evolved in Oxygenation of Blood. Conditions of Oxygen and of Blood that modify Combination. Modifications of Oxygen-Ozone. Influence of Temperature.

I REFERRED in my last chapter to the two modes, external and internal, by which force or motion is communicated to the body. When we view the subject free from all prejudice and all superstition, we are driven to the conclusion that by none other than the means there described can motion be supplied: To presume that the nervous system can generate motion, that it can out of nothing produce something, is like saying that a galvanic battery may work a telegraph without chemical action. The nervous system is the centre of forces derived from without and within-the tabula rasa on which the external world is written - the centre in which motion derived from the blood is laid up, to be applied in volition. But, beyond this, the nervous system is not different from other parts of the physical organism.*

Let us then consider the source of that motion which springs from within the body; which, in better words, is evolved in the body; which gives animal power, but does not give intelligence; which makes the animal an engine, but not an animal; which is constructive and locomotive, but not animating ; but which yet prepares the machine for animation, and without which there is no life.

. This force or motion is elicited by the combination of the oxygen of the air with the carbon derived from the vegetable kingdom, and present in solution or suspension in the blood. We speak of the force thus evolved as primary in respect to the body, and so it is; but, in truth, we may trace it back further-at least, to the sun.

When the sunbeam falls on the vegetable world, when the radiant motion from the sun touches the vegetable world, that chemical change takes place in the leaf of the plant which is described as consisting of the decomposition of carbonic acid, the evolution of oxygen, and the fixation of carbon. This is the chemical part of the change that takes place; but there is a physical part played as well. The motion derived from the sun is also fixed, to be laid by until it is required, until the time has come when, by the recombination of oxygen with carbon, it shall be set at liberty in the form of heat, as heat of fire, or heat of body. Thus the force that supplies the organism with the motion that pertains strictly to its organic or vegetative life is conveyed into the body as coal is conveyed to the fire, through the vegetable worldconveyed as fuel to the furnace, and as food to the animal. Plants are condensers of motion for man; they take up from the earth the dead matter; they take from the sun motion; they endow the dead matter with motion; and, thus endowing it, they become to the animal constructively and actively as matter and motion.

When distributed through the blood, the carbon from the plant meets, in infinite subdivision of mole-

* In a paper read before the Medical Society of Iondon in the beginning of 1863 , I described the nervous system as the condenser of motion. 